\title{
Review of: "A naturally DNase-free CRISPR-Cas12c enzyme silences gene expression"
}

\author{
Fatimah Aljedaani ${ }^{1}$ \\ 1 King Abdullah University of Science and Technology
}

Potential competing interests: The author(s) declared that no potential competing interests exist.

This manuscript by Jennifer A. Doudna et al. does an excellent job by harnessing DNase-free CRISPR-Cas (Cas12c) as a system to target silencing gene expression and to provide antiviral immunity in bacteria without target-activated DNase activity. The authors have clarified the ability of Cas12c as a ribonuclease; it cannot cleave DNA targets but can bind to the DNA targets.

The authors provided evidence and details confirming that despite the lack of DNA targeting cleavage, Cas12c binds to target DNA through a canonical crRNA-mediated interaction and mediates robust repression of target transcription. Furthermore, the authors have shown that Cas12c could protect cells from bacteriophage infection as DNA-targeting CRISPR ribonuclease without DNase activity.

In summary, the manuscript is well-written and nicely demonstrated the potential of CRISPR/ Cas12c to target gene silencing and antivirus immunity via binding but not through cutting DNA sequence. This work may add a significant contribution to develop CRISPR/Cas-based technologies for gene silencing and antiviral approach. 